\title{
Anticorps et bioterrorisme
}

Philippe Thullier, Thibaut Pelat, Dominique Vidal

> Le risque d'utilisation d'armes biologiques pourrait conduire à la réémergence de graves pathologies, telles que la peste ou la variole, maintenant éradiquées des pays développés. La prise en charge de ce risque, appelé risque biologique provoqué (RBP), comporte notamment le développement d'anticorps recombinants - qui est une voie assez sûre pour obtenir des molécules bien tolérées pour la prévention et le traitement des maladies humaines - parce que les anticorps neutralisent de nombreux agents pathogènes. Des travaux récents ont ainsi permis d'isoler des anticorps recombinants neutralisant la toxine létale du charbon, les toxines botuliques et le virus de la variole en particulier, grâce à différentes technologies. Notre approche, consistant à obtenir des anticorps recombinants en construisant des librairies immunes exposées à la surface de phages à partir de primates non humains (M. fascicularis), puis à humaniser ces anticorps par ingénierie, nous permet de participer efficacement à ces travaux. <

\section{Risque biologique provoqué : interêt des anticorps}

Le bioterrorisme et l'utilisation d'armes biologiques forment respectivement les versants civil et militaire du risque biologique provoqué (RBP). On désigne sous ce terme la dissémination de virus, bactéries ou toxines pour agresser des populations civiles ou des troupes en opération. Des enveloppes contenant des spores du charbon ont contaminé des patients aux États-Unis en 2001 [1] et ont bruyamment rappelé la réalité de ce risque, mais plusieurs autres exemples, plus anciens [2] ou plus récents [3], en témoignent également. Ainsi, si l'utilisation des armes biologiques est interdite par le droit international, les formes non conventionnelles de la guerre sont trop imprévisibles pour ne pas chercher à prévenir ce

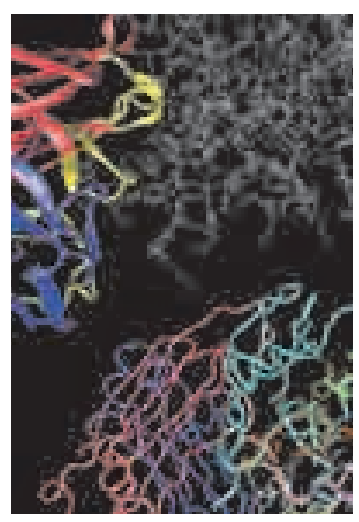

P. Thullier, T. Pelat, D. Vidal : Groupe de biotechnologie des anticorps, département des agents transmissibles, Centre de recherche du Service de santé des armées, 38702 La Tronche Cedex, France. pthullier@yahoo.com.

risque. Le charbon, la peste, la variole, la mélioïdose $^{1}$ et le botulisme sont maintenant quasiment éradiqués des pays développés par des mesures de santé publique et d'hygiène, mais sont les principaux candidats du RBP, de même que l'intoxication par la ricine ${ }^{2}$. Le nombre d'agents potentiellement impliqués (par exemple, certaines fièvres hémorragiques et encéphalites virales font partie du RBP) est toutefois plus grand. La surveillance des sources d'alimentation limite l'exposition des militaires mais plus difficilement celle des populations civiles, et tous sont exposés au risque de contamination par voie pulmonaire (inhalation). Les vaccins protègent souvent en induisant la synthèse d'anticorps ( $A C$ ) neutralisants, mais des programmes de vaccination protégeant contre d'aussi nombreux agents sont difficiles à mettre en œuvre, même chez les militaires dont tous ne seront pas exposés au RBP au cours de leur vie professionnelle. La mise en place de tels programmes au profit des populations civiles est encore plus difficile à concevoir. Les Ac permettent la neutralisation d'un grand nombre d'agents du RBP et l'administration d'Ac préformés présente l'avantage d'apporter une prophylaxie immédiatement efficace, qui peut donc être mieux ciblée sur les personnels considérés comme effectivement exposés (par

${ }^{1}$ La mélioïdose est une zoonose bactérienne, endémique en Asie, due à un bacille tellurique, Burkholderia pseumallei ou bacille de Whitmore, qui contamine de nombreux mammifères domestiques. C'est une infection sévère, à mortalité élevée, qui se manifeste sous trois formes : septicémique, viscérale localisée et latente.

${ }^{2}$ La ricine est une toxine qui se trouve dans les graines de Ricinus comminis. Ces plantes sont cultivées partout dans le monde, pour produire l'huile de ricin ou dans un but ornemental. 
exemple, personnels de premiers secours ou personnes fréquentant des endroits symboliques du pays en crise). Le niveau de protection, corrélé à la concentration circulante d'Ac neutralisants, peut être supérieur à celui apporté par la vaccination et sa durée est déterminée par la demi-vie des anticorps qui est d'un mois environ. L'utilisation thérapeutique des Ac est aussi efficace, sous réserve d'une administration assez rapide (de l'ordre de plusieurs heures, le plus souvent) après exposition à l'agent considéré.

\section{Stratégies d'obtention et d'optimisation des anticorps recombinants contre les agents pathogènes du RBP}

Les Ac recombinants, ainsi appelés parce qu'ils sont obtenus après manipulation et expression d'ADN, et non plus extraits de produits sanguins, forment une classe thérapeutique récemment apparue et en très forte expansion. Ces molécules sont capables de neutraliser spécifiquement leurs cibles grâce à plusieurs mécanismes. Les Ac existent naturellement dans l'organisme et leur bonne tolérance, sous réserve qu'ils soient d'origine humaine ou au moins offrant une grande similitude avec leurs homologues humains, peut expliquer pourquoi leur développement peut être préféré à celui des petites molécules synthétiques dont 9 sur 10 échouent lors des essais cliniques [4], précisément en raison d'un défaut de biodisponiblité ou d'induction d'effets secondaires sévères. Les anticorps seraient utilisés en association aux médicaments éventuellement disponibles dont les mécanismes d'action sont souvent très différents, créant ainsi une synergie bénéfique. Des IgA (immunoglobulines $A$ ) pourraient être préférées aux IgG puisque les deux modes de contamination envisagés (inhalation et absorption par voie orale) supposent le passage à travers des muqueuses, site privilégié d'action de ces IgA.

Les $A c$ recombinants sont généralement obtenus en deux étapes: obtention d'un fragment d'Ac qui se fixe à l'agent devant être neutralisé, puis synthèse d'une IgG entière à partir de ce fragment. Des fragments d'Ac recombinants se fixant sur l'une des sous-unités de la toxine létale du charbon, appelée antigène protecteur, ont été obtenus par plusieurs équipes dont la nôtre [5-11]. Ils pourraient prévenir ou traiter cette maladie plus efficacement que les antibiotiques seuls. Ces derniers agissent en effet sur la septicémie en tuant les bactéries végétatives mais ne peuvent pas agir sur les toxines qui ont été préalablement libérées par ces bactéries lors de leur phase de multiplication. Le fragment d'Ac obtenu dans notre laboratoire a été exprimé sous forme d'IgG, et ses capacités de protection prophylactiques et thérapeutiques ont été vérifiées dans un modèle animal de la maladie. Nous avons également obtenu un fragment d'Ac recombinant dirigé contre la seconde sous-unité de cette même toxine, le facteur létal [12], comme cela avait été préconisé par les experts du charbon [13]. La mélioïdose [14], et surtout la peste [15], sont des maladies bactériennes incluses dans le RBP que des Ac peuvent aider à contrôler. Le virus de la variole peut être neutralisé par des Ac dirigés contre les protéines de surface B5 [16] ou L1 [17]. La neutralisation des toxines botuliques - substances qui sont de loin les plus toxiques connues [18] - est possible par des Ac dont les caractéristiques sont main- tenant bien décrites. Nous avons obtenu un fragment d'Ac neutralisant la ricine dont l'utilisation optimale se ferait probablement par aérosol [19].

Les fragments d'anticorps recombinants (Fab ou $\mathrm{scFv})(\rightarrow)$ peuvent être obtenus en fabriquant et en criblant des librairies de fragments d'Ac $(\rightarrow)$ voir $R$. Abès et al., page 1011 ; P. Chames et D. Baty, page 1159 exposés à la surface de phages [20], ce qui est la stratégie la plus robuste utilisée dans ce domaine de recherche. Toutefois, cette stratégie est plus efficace lorsqu'elle débute en utilisant des lymphocytes B de donneurs humains immunisés contre l'agent d'intérêt. De telles cellules sont disponibles aux États-Unis dans le cas du charbon [8] puisque la prévention de cette maladie est obtenue chez les militaires par une vaccination qui repose sur l'utilisation de l'antigène protecteur $(A P)^{3}$ comme immunogène. Dans d'autres cas, comme le botulisme, des essais de vaccination ont permis d'obtenir de tels lymphocytes [21]. Des chimpanzés, dont les anticorps sont identiques aux anticorps humains et qui avaient été utilisés aux États-Unis pour des essais de vaccination contre la variole, ont été revaccinés puis leurs cellules utilisées de la même façon [22]. En Europe toutefois, l'utilisation des chimpanzés au laboratoire est quasiment interdite, pour des raisons éthiques. Des souris dont les locus codant les immunoglobulines sont remplacés par leurs homologues humains ont pu être utilisées [23-25] $(\rightarrow)$, mais cette technique est délicate et $\rightarrow$ voir M. Cogné et al., page 1149 très coûteuse. D'une façon générale, la rareté dans nos pays des maladies naturellement provoquées par les agents recensés dans le RBP, et l'absence de vaccination contre ce risque, nous ont empêché d'avoir accès à des lymphocytes de sujets immunisés contre ces pathogènes. Dans notre laboratoire, les librairies ont donc été obtenues en partant de macaques que nous avons immunisés, ce qui est une approche peu fréquente mais justifiée par la très grande similitude existant entre les fragments d'anticorps de primates non humains (ici Macaca fascicularis) et ceux de l'homme [26]. Cette approche permet de cibler très précisément les protéines essentielles impliquées dans la physiopathologie de la maladie en les utilisant comme immunogènes, ce qui ne serait pas possible chez le sujet humain. De plus, les lymphocytes peuvent être obtenus à partir de la moelle osseuse, ce qui présente des avantages pour notre technique alors que ce tissu est beaucoup plus difficilement accessible chez l'homme pour des raisons éthiques. Pour compléter notre approche et en utilisant

${ }^{3}$ On appelle antigène protecteur - paradoxalement - une sous-unité des toxines du charbon initialement identifiée pour ses propriétés vaccinales. 
un site d'analyse de séquences (http://imgt.cines.fr) [27] $(\rightarrow)$, puis un site de modélisation tridimensionnelle des Ac (http://antibody.bath.ac.uk)

$(\rightarrow)$ voir M.P. Lefranc, page 1020

[28], nous avons pu muter un premier fragment d'Ac de macaque pour le rendre «plus humain » - très exactement, pour le rendre plus proche des séquences germinales humaines (gènes $V$, (D), J non réarrangés) codant des Ac qu'un Ac issu de tel ou tel donneur humain particulier [29]. Cette technique d'ingénierie des Ac, qui en préserve l'affinité pour leur cible, est en cours d'application à l'ensemble des fragments d'Ac obtenus dans notre laboratoire dans le but d'en assurer une excellente tolérance [2]. De façon générale, les travaux réalisés dans notre laboratoire sont susceptibles d'améliorer significativement la prise en charge prophylactique et thérapeutique des agents à l'origine du RBP, ce d'autant qu'il existe en France une voie particulière d'agrément des médicaments ciblant spécifiquement ce risque, auprès des autorités de santé. La versatilité de l'approche utilisée ne la cantonne évidemment pas à la prise en charge du RBP et elle est aussi applicable aux pathologies rencontrées en pratique plus courante. $\diamond$

\section{SUMMARY}

Recombinant antibodies against bioweapons The threat posed by bioweapons (BW) could lead to the re-emergence of such deadly diseases as plague or smallpox, now eradicated from industrialized countries. The development of recombinant antibodies allows tackling this risk because these recombinant molecules are generally well tolerated in human medicine, may be utilized for prophylaxis and treatment, and because antibodies neutralize many BW. Recombinant antibodies neutralizing the lethal toxin of anthrax, botulinum toxins and the smallpox virus have in particular been isolated recently, with different technologies. Our approach, which uses phage-displayed immune libraries built from non-human primates (M. fascicularis) to obtain recombinant antibodies, which may later be super-humanized (germlinized), has allowed us to obtain such BWsneutralizing antibodies. $\diamond$

\section{CONFLIT D'INTÉRÊTS}

Les auteurs déclarent n'avoir aucun conflit d'intérêts concernant les données publiées dans cet article.

\section{RéFÉRENCES}

1. Inglesby TV, O'Toole T, Henderson DA, et al. Anthrax as a biological weapon, 2002: updated recommendations for management. JAMA 2002 ; $287: 2236-52$

2. Binder P, Lepick O. Les armes biologiques. Paris : PUF, 2001.
3. Mayor S. UK doctors warned after ricin poison found in police raid. Br Med J $2003 ; 326: 126$.

4. Kola I, Landis J. Can the pharmaceutical industry reduce attrition rates? Nat Rev Drug Discov $2004 ; 3: 711-5$.

5. Sawada-Hirai R, Jiang I, Wang F, et al. Human anti-anthrax protective antigen neutralizing monoclonal antibodies derived from donors vaccinated with anthrax vaccine adsorbed. J Immune Based Ther Vaccines $2004 ; 2: 5$.

6. Little SF, Leppla SH, Cora $\varepsilon$. Production and characterization of monoclonal antibodies to the protective antigen component of Bacillus anthracis toxin. Infect Immun 1988; $56: 1807-13$.

7. Little SF, Ivins BE, Fellows PF, Friedlander AM. Passive protection by polyclonal antibodies against Bacillus anthracis infection in guinea pigs. Infect Immun 1997 ; 65 : 5171-5.

8. Wild MA, Xin H, Maruyama T, et al. Human antibodies from immunized donors are protective against anthrax toxin in vivo. Nat Biotechnol $2003 ; 21$ : 1305-6.

9. Mohamed N, Clagett M, Li J, et al. A high-affinity monoclonal antibody to anthrax protective antigen passively protects rabbits before and after aerosolized Bacillus anthracis spore challenge. Infect Immun 2005; 73: 795-802.

10. Wang F, Ruther $P$, Jiang I, et al. Human monoclonal antibodies that neutralize anthrax toxin by inhibiting heptamer assembly. Hum Antibodies $2004 ; 13: 105-10$.

11. Laffly $\varepsilon$, Danjou L, Condemine F, et al. Selection of a macaque Fab with framework regions like those in humans, high affinity, and ability to neutralize the protective antigen (PA) of Bacillus anthracis by binding to the segment of PA between residues 686 and 694 . Antimicrob Agents Chemother $2005 ; 49: 3414-20$.

12. Pelat $T$, Hust $M$, Laffly $\varepsilon$, et al. High-affinity, human antibody-like antibody fragment (singlechain variable fragment) neutralizing the lethal factor (LF) of Bacillus anthracis by inhibiting protective antigen-LF complex formation. Antimicrob Agents Chemother 2007 ; 51 : 2758-64.

13. Baillie LSL, Quinn C, Swann P, et al. Expert Consultation on Monoclonal Antibodies for Anthrax rPA. 2003. Online: http://www3.niaid.nih.gov/biodefense/resaerch/products.htm\#5.

14. Jones SM, Ellis JF, Russell P, et al. Passive protection against Burkholderia pseudomallei infection in mice by monoclonal antibodies against capsular polysaccharide, lipopolysaccharide or proteins. J Med Microbiol 2002 ; 51 : 1055-62.

15. Hill J, Eyles JE, Elvin SJ, et al. Administration of antibody to the lung protects mice against pneumonic plague. Infect Immun $2006 ; 74: 3068-70$.

16. Chen Z, Earl P, Americo J, et al. Chimpanzee/human mAbs to vaccinia virus B5 protein neutralize vaccinia and smallpox viruses and protect mice against vaccinia virus. Proc Natl Acad Sci USA $2006 ; 103: 1882-7$.

17. Su HP, Golden JW, Gittis AG, et al. Structural basis for the binding of the neutralizing antibody, 7D11, to the poxvirus L1 protein. Virology 2007 ; 368 : 331-41.

18. Arnon SS, Schechter R, Inglesby TV, et al. Botulinum toxin as a biological weapon: medical and public health management. JAMA $2001 ; 285$ : 1059-70.

19. Foxwell BM, Detre SI, Donovan TA, Thorpe PE. The use of anti-ricin antibodies to protect mice intoxicated with ricin. Toxicology $1985 ; 34: 79-88$.

20. Dubel S. Handbook of therapeutic antibodies. Weinheim : Wiley-VCH, 2007.

21. Amersdorfer P, Wong C, Smith T, et al. Genetic and immunological comparison of antibotulinum type $A$ antibodies from immune and non-immune human phage libraries. Vaccine $2002 ; 20: 1640-8$.

22. Chen Z, Earl P, Americo J, et al. Characterization of chimpanzee/human monoclonal antibodies to vaccinia virus $A 33$ glycoprotein and its variola virus homolog in vitro and in a vaccinia virus mouse protection model. J Virol $2007 ; 81: 8989-95$.

23. Choi TK, Hollenbach PW, Pearson BE, et al. Transgenic mice containing a human heavy chain immunoglobulin gene fragment cloned in a yeast artificial chromosome. Nat Genet 1993; $4: 117-23$.

24. Fishwild DM, O'Donnell SL, Bengoechea T, et al. High-avidity human IgG kappa monoclonal antibodies from a novel strain of minilocus transgenic mice. Nat Biotechnol 1996 ; $14: 845-51$.

25. Bellet D, Pecking A, Dangles-Marie V. XenoMouse : un tour de force pour l'obtention d'anticorps humains chez la souris. Med Sci (Paris) 200824 : 903-5.

26. Barbas CF. Phage display: a laboratory manual. New York : Cold Spring Harbor Laboratory Press, 2001.

27. Brochet X, Lefranc MP, Giudicelli V. IMGT/V-QUEST: the highly customized and integrated system for IG and TR standardized V-J and V-D-J sequence analysis. Nucleic Acids Res 200836 : W503-8.

28. Whitelegg NR, Rees AR. WAM: an improved algorithm for modelling antibodies on the WEB. Protein Eng $2000 ; 13: 819-24$.

29. Pelat $T$, Bedouelle $H$, Rees AR, et al. Germline humanization of a non-human primate antibody that neutralizes the anthrax toxin, by in vitro and in silico engineering. J Mol Biol 2008; $384: 1400-7$ 


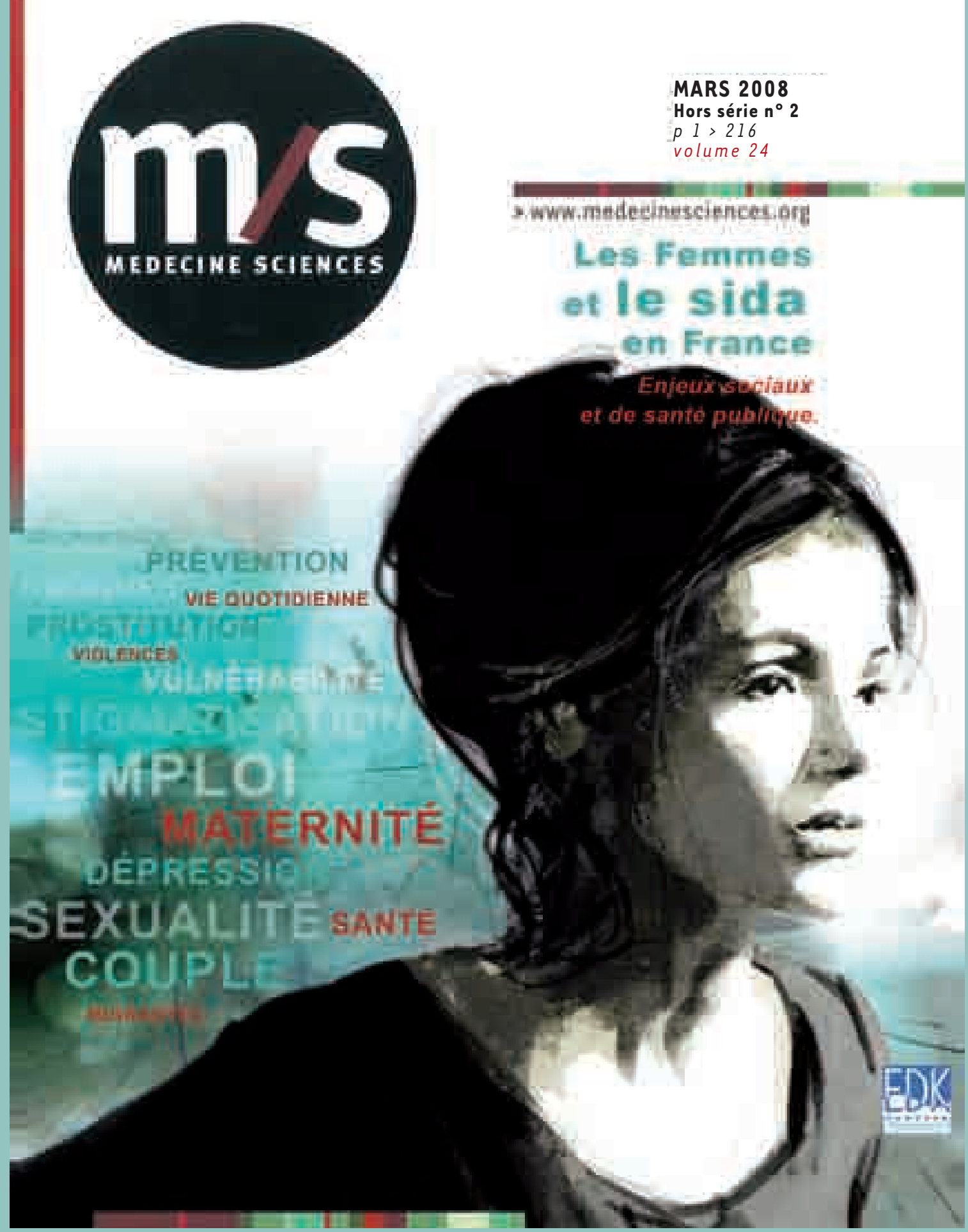

L'Anrs (Agence nationale de recherches sur le sida et les hépatites virales) publie « Les femmes et le sida en France - Enjeux sociaux et de santé publique ». Un numéro indispensable pour comprendre les nouveaux enjeux de prévention dans le contexte de féminisation et de précarisation de l'épidémie et au moment où se pose la question des conditions de vie des femmes atteintes par l'infection du VIH en France.

Un ouvrage précieux, destiné aux chercheurs, élus, décideurs en santé et représentants associatifs.

\section{Bon de commande}

À retourner à EDK, 2, rue Troyon - 92316 Sèvres Cedex

Tél. : 0155641393 - Fax : 0155641394 - E-mail : edk@edk.fr

NOM : Prénom :

Adresse :

Code postal : Ville :

Pays :

Fonction :

Je souhaite recevoir le hors série ${ }^{\circ} 2$ de $M / S$ Les femmes et le Sida en France : $18 €+3 €$ de port $=21 €$ TTC

en ................ exemplaire, soit un total de

$\square$ Par chèque, à l'ordre de $\mathbf{E} \mathbf{D} \mathbf{K}$

$\square$ Par carte bancaire : $\square$ Visa $\square$ Eurocard/Mastercard

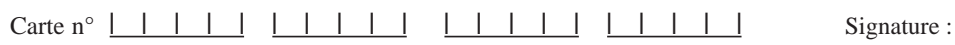

Date d'expiration: $\quad \underline{1 \quad 1 \quad 1 \quad 1 \quad 1}$

$N^{\circ}$ de contrôle au dos de la carte : $\quad \underline{\text { । }}$ 